\title{
Efficient GTS Allocation Algorithm for IEEE 802.15.4
}

\author{
Youngmin $\mathrm{Ji}^{1}$, Woojin $\mathrm{Park}^{2}$, Sungjun $\mathrm{Kim}^{2}$, and Sunshin $\mathrm{An}^{2}$ \\ ${ }^{1}$ Department of Telecommunication System Technology, Korea University, \\ 5Ga 1, Anamdong, Sungbukku, Seoul 136-701, Republic of Korea \\ ymji@dsys.korea.ac.kr \\ ${ }^{2}$ Department of Electronics and Computer Engineering, Korea University, \\ 5Ga 1, Anamdong, Sungbukku, Seoul 136-701, Republic of Korea \\ \{wjpark,sjkim, sunshin\}@dsys.korea.ac.kr
}

\begin{abstract}
Recently issued standard IEEE 802.15.4 is a novel standard to achieve some critical features of wireless sensor networks. In this paper, we propose the algorithm to allocate GTSs according to the packet arrival rate from end devices and the number of devices in beacon enabled mode of IEEE 802.15.4 star topology networks. From simulation results, we show that our proposed algorithm enhances throughput comparing with the original IEEE 802.15.4 that uses only CAP duration for transmission $^{1}$.
\end{abstract}

Keywords: IEEE 802.15.4, Wireless Sensor Networks, GTS, Zigbee.

\section{Introduction}

In this paper, we introduce the algorithm to enhance network throughput by allocating GTSs efficiently in beacon enabled mode of IEEE 802.15.4 star topology networks. We use ns-2 simulations to prove our proposed algorithm. The simulation is performed by implementing our algorithm and GTS module in IEEE 802.15.4 of ns-2 3] [4. Our simulation results indicate that proposed algorithm improves throughput comparing with the original IEEE 802.15.4.

\section{Efficient GTS Allocation Algorithm}

In this section, we present the proposed algorithm to allocate efficiently GTSs based on the information from end devices in IEEE 802.15.4 star topology networks. The information is the packet arrival rate and the number of devices. They are selected as the criteria of our algorithm due to follows: 1) Packet arrival rate: the devices with the higher packet rate can cause the more collisions and the much delay compared to devices with the lower packet rate during trying to transmit their data packets. Allocating GTSs to devices with the higher packet rate can minimize packet collisions and delay of the network. 2) Number

\footnotetext{
${ }^{1}$ This research was partly supported by SK Telecom (SKT) in Korea.
} 
of devices: GTSs are designed to be used at ranges less than 8 slots in IEEE 802.15.4 1]. Therefore, the coordinator can allocate the maximum 7 GTSs in the range of 0 through 6 . Based on the criteria, our algorithm allocates one GTS slot to each node in order of high packet arrival rate and the maximum number of GTS allocation $\left(n_{G}\right)$ depends on the size of the network as shown in (11).

$$
n_{G}=\left\llcorner\frac{n}{2}\right\lrcorner \quad(\text { if } n \leq 15), \quad n_{G}=7 \quad(\text { if } n>15)
$$

In this algorithm, a coordinator manages each end device's $T_{C A}$ (Current Arrival Time), $T_{P A}$ (Previous Arrival Time), $T_{C I}$ (Current Interarrival Time), $T_{A I}$ (Average Interarrival Time) and $\delta$ (Parameter to control the relative weight of recent and past interarrival rate). If $\delta$ is $0, T_{A I}$ is ignored. If $\delta$ is $1, T_{C I}$ cannot affect $T_{A I}$. We have to choose $\delta$ at ranges of larger than 0 and less than 1 . We set $\delta$ as $0.9, T_{A I}$ is more weighted than $T_{C I}$ (Note: $T_{A I j}=T_{A I j} \delta+T_{C I j}(1-\delta)$ of Algorithm 1) since data packet is frequently received. The detailed sequence of the proposed algorithm is described in Algorithm 1, which runs in the coordinator.

\section{Algorithm 1: Record Interarrival Time}

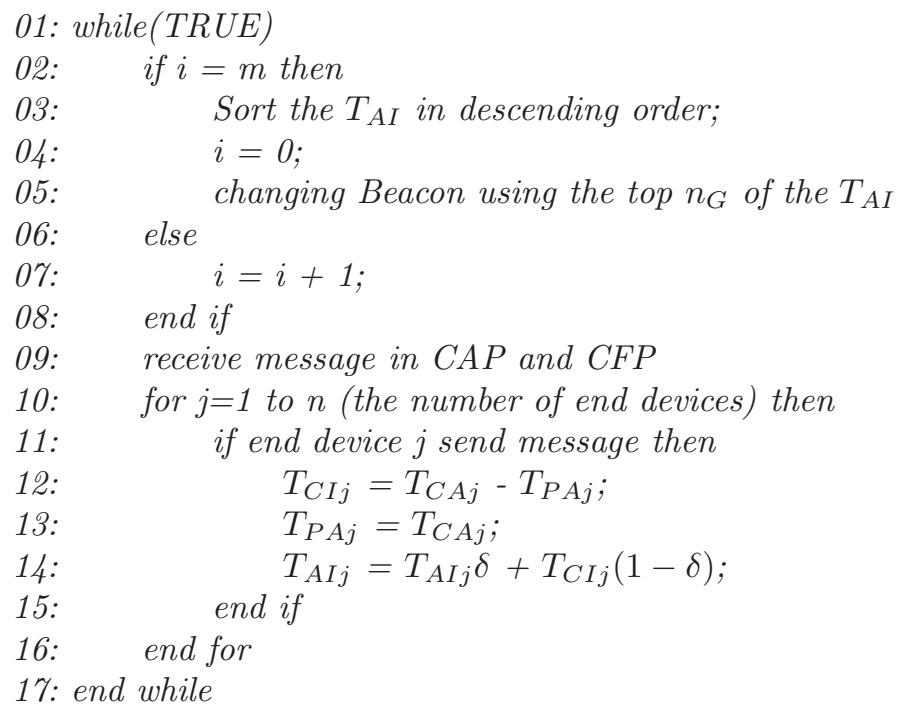

\section{Simulation}

\subsection{Simulation Environment}

The network is a star topology that consists of 17 devices containing a PAN coordinator. We set BO (Beacon Order) and $S O$ (Superframe Order) to 7 so that coordinator and end devices are always in active mode and never switch to sleep mode since we do not need to use the inactive periods. Simulations are conducted with three groups that consist of Group 1 (Device 1 to 6), Group 2 
(Device 7 to 11 ) and Group 3 (Device 12 to 16). Each group has the different transmission rate $(1,0.01$ and 0.1 in order of Group) to show that GTSs are dynamically allocated in order of transmission rates. The size of a transmitted packet is 80 bytes. The transmission rate of a device is $240 \mathrm{kbps}$.

\subsection{Simulation Results}

Fig 1 represents the history of allocating GTSs on each device. This result indicates that the occupation rate of time slots depends on the traffic rate. We can know the amount of successful transmissions on each device, which use the guaranteed time slots.

Fig 2 shows the comparison of packet delivery and packet drop. As expected, the average throughput of IEEE 802.15 .4 is up to $168 \mathrm{kbps}$; however the average throughput of our proposed algorithm is up to $195 \mathrm{kbps}$. We can get $16 \%$ improvement in throughput in simulation. By using this result, we can understand the efficiency of proposed scheme.

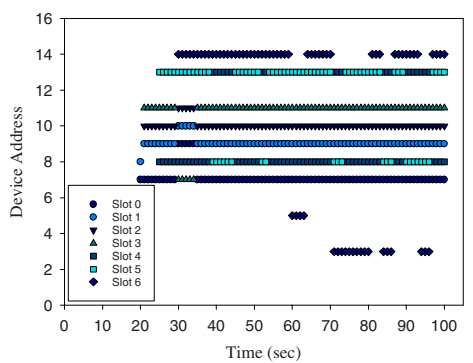

(a) History of GTS allocation

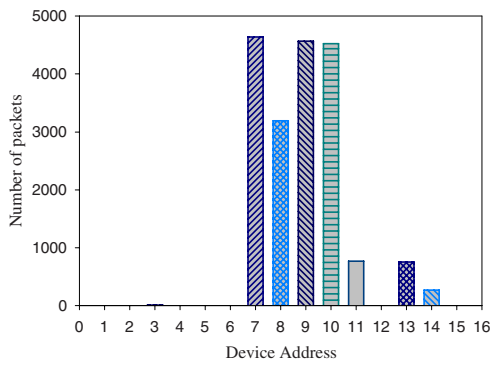

(b) Successful Packet Transmission

Fig. 1. GTS allocation and successful transmission on each device with our algorithm
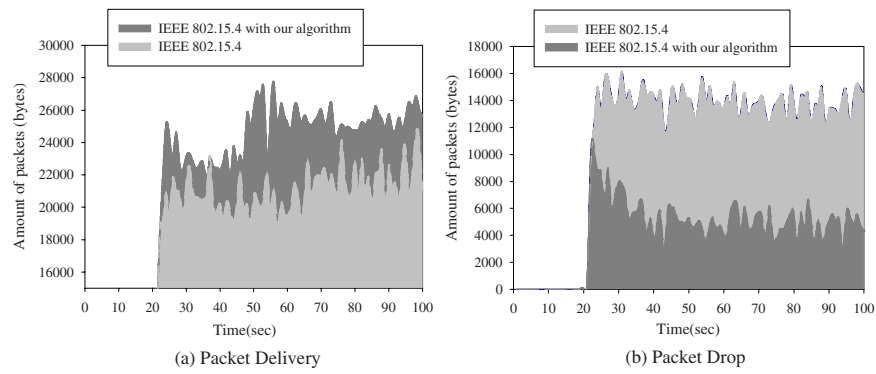

Fig. 2. Comparison of packet delivery and drop number

In addition, when the device employs IEEE 802.15.4 with our algorithm, the amount of drop packets caused by a lot of collisions decreases as shown in Fig, 2 The average packet drop rate of IEEE 802.15.4 is up to $110 \mathrm{kbps}$ but average 


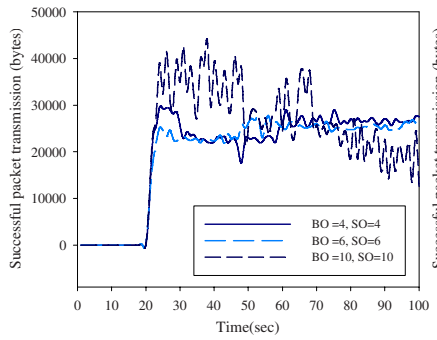

(a) IEEE 802.15.4 with our algorithm

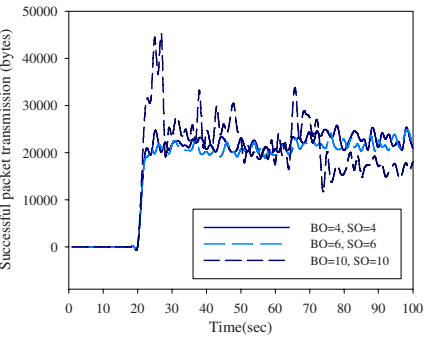

(b) IEEE 802.15 .4

Fig. 3. Comparison of successful transmission on various parameters

packet drop rate of our proposed algorithm is up to $43 \mathrm{kbps}$. This result also indicates that our proposed algorithm is more efficient than existing protocol. Fig 2 and Fig 3 represent the throughput of IEEE 802.15.4 with our algorithm and IEEE 802.15.4 with various parameters respectively. In a case of IEEE 802.15.4 with our algorithm, the average throughput is up to $206 \mathrm{kbps}$. In a case of IEEE 802.15.4, the average throughput is up to $174 \mathrm{kbps}$. While changing several parameters we have obtained better results through the IEEE 802.15.4 with our algorithm. Finally, we can get $18 \%$ improvement in average throughput in these scenarios.

\section{Conclusion}

In this paper, an efficient GTS allocation algorithm was proposed to guarantee the throughput for sensor devices and proved through the simulation. Our proposed algorithm showed the improved throughput comparing with the original IEEE 802.15.4 that uses only CAP duration for transmission. This algorithm is adaptable not only star topology but also multi-hop topology for beacon enabled mode.

\section{References}

1. IEEE Standard for Wireless Medium Access Control (MAC) and Physical Layer (PHY) Specification for Low-Rate Wireless Personal Area Networks (LR-WPAN), Oct. 2003

2. Z.Alliance, "Zigbee specification v1.0," June 2005

3. USC Information Sciences Institute, Marina del Rey, CA. Network Simulator - ns-2. (http://www.isi.edu/nsnam/ns)

4. Samsung/CUNY, "ns-2 simulator for IEEE 802.15.4," http://www-ee.ccny.cuny.edu/zheng/pub [referenced September 13, 2005] 